\title{
A Review on Thinning in Digital Image Processing
}

\author{
Preeti Bansal ${ }^{1}$, Balwinder Kaur ${ }^{2}$ \\ ${ }^{1}$ Student - Department of Computer Science and Engineering, Lovely Professional University, Punjab, India \\ ${ }^{2}$ Assistant Professor - Department of Computer Science and Engineering, Lovely Professional University, Punjab, India
}

\begin{abstract}
Thinning is the first step which we can say that "Pre-processing". This reduction may be accomplished by obtaining the skeleton of the region using skeletonization also known as thinning. There were many algorithms which were developed to implement thinning algorithm but all were not able to give the successful output because of not fulfilling the basic requirements which are the sensitivity to noise and reconstruct-able. There are two types of thinning which are iterative and non-iterative. These all algorithm can be implemented by various software's like MATLAB and Verilog. These all algorithm performance can be measured by one factor thinning rate. So, there is need to propose new algorithm which will give efficient results in terms of thinning rate, thinning speed, number of connected components, PSNR(peak signal to noise ratio), MSE(Mean square Value) etc.
\end{abstract}

Keywords: Skeletonization, Thinning Rate (TR)

\section{Introduction}

Digital Image Processing comprises of three words: Digital, Image \& Processing. It works on the images with take digital image as the input side works on the noise reduction, signal distortion and give us the output.This works on the system and signals but mainly works on the images. It apply efficient algorithm to give as image as the output. We compare that output with our desired output if it does not match that output we give it as an output until and unless we get our output as required or approximately value. Digital image processing is far better than analog signals as it does not work on multi-dimensional pixels. Digital image is an array or a matrix represented by a finite number of bits.

\subsection{Introduction to Thinning}

One of the fundamental requirements is to represent the structural shape of digital images. This can be done by reducing it to a graph. This reduction may be accomplished by obtaining the skeleton of the region using skeletonization also known as thinning. Thinning is the first step which we can say that "Pre-processing". We can redefine the thinning which is extraction of skeleton or reducing an digital image to the minimum size or to reduce the image up to this extent so that image preserves the points need for image processing. We can Evaluate the performance on the basis of following parameters:- Processing time, connectivity ,image should not contain noise and branches. There were many algorithms which were developed to implement thinning algorithm but all were not able to give the successful output because of not fulfilling the basic requirements.

Senstivity to Noise:-It means that the image should not contain that pixels which will help to produce incorrect output. We can say that if there will be noise that after applying the algorithm we will not able to get the desired output.

Reconstructable:- According to this if we apply any algorithm on to the thinned image we must get the output as thinned image or original image. Skeletonization is usually applied on binary images which consist of black (foreground) and white (background) pixels. It takes input a binary image, and produces another binary image

\subsection{Types of Thinning}

Iterative thinning algorithms:- It works on the pixel by pixel based thinning. It examine the pixels until the result is obtained. It mainly divides into two parts Parallel and sequential.

Sequential thinning takes place in predetermined order in which processing takes place in fixed sequence. There is mainly one difference between these two sequential depends upon previous iteration result and also all the iterations done till now.

But in parallel thinning only the result that remains after the previous iteration is taken in consideration.

Directional approach: - It was first done by Rosenfeld in 1970. In this thinning is done with respect to the directions that are north, south, east west. In these points belonging in the same side is removed parallel.

Sub-field approach:-In this image is sub divided into parts according to some criteria.There is no some fixed criteria of dividing but some parity criteria is applied on it. All the pixels in one direction is removed at one time.

Fully parallel approach:- In this neighbourhood criteria is used. The image can be divided in any format either in $\mathrm{k} * \mathrm{k}$ or $n * k$ Example:- $3 * 3$ or $4 * 3$ or $3 * 4$.

Non-Iterative Algorithm:- It is just opposite to iterative as it does not works on the pixel to pixel examine. There are some popular methods on which implementation is based like medical transforms, distance transforms and other methods.

Medical Transforms:- It results do contain noise in data as it works on the technique of grey level images where intensity is represented in the terms of distance to the boundary 


\section{International Journal of Science and Research (IJSR) \\ ISSN (Online): 2319-7064 \\ Index Copernicus Value (2013): 6.14 | Impact Factor (2015): 6.391}

Central line: It takes the minimum time to process as it takes a center line or median for processing and thinning is done in one process or at one time. And there is one also form that distance to the boundary which help us to know whether this point should be considered in the skeleton or not

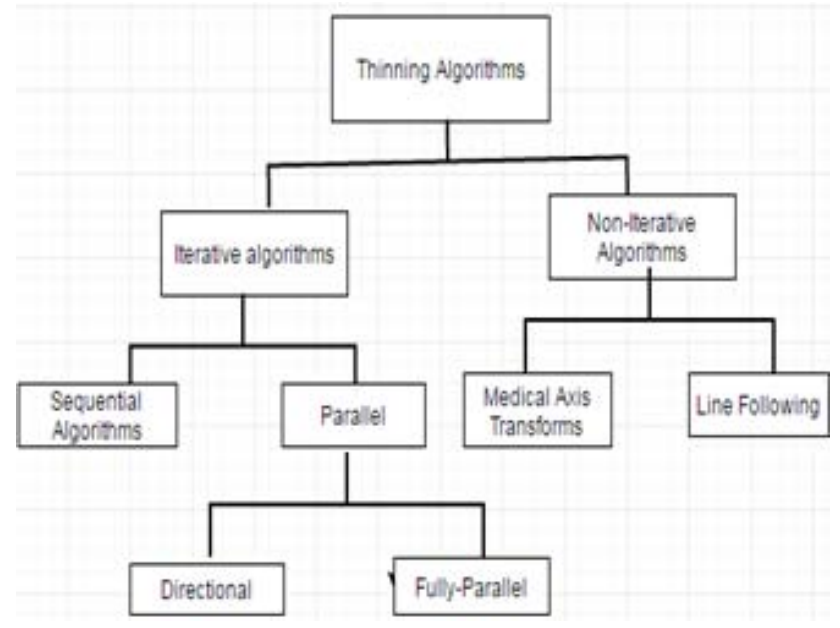

\subsection{Various Algorithms in Thinning}

There are various algorithms to implement all these concepts:-

1) Zhang Suen Thinning algorithm.

2) Canny Edge detection.

3) Edge Based Thinning algorithm.

4) Optimized iterative algorithm using successive erosion.

5) Guo and Hall's parallel Thinning algorithm.

\subsection{Need of Skeletonization}

Therefore, it is an wide area of research to improve the results as much as possible So, there is always a need for good skeletonization algorithms in reference to following parameters:

1) To reduce processing time.

2) By reducing an object to only a skeleton, unimportant features and image noise can be filtered out.

3) If we remove extra points it will take less time for data processing and handling of database will be easy.

\section{Literature Survey}

[1] In this the author describes new two pass parallel algorithm for the binary images. The algorithm makes the image to one pixel thick width and preserves the connectivity of components. This algorithm also helps to preserve 8 neighbour connectivity in binary images. The proposed algorithm shows better performance in terms of connectivity and one pixel thick and produces high quality images than the previous Skeletonization algorithms. Experimental results shows the advantage of proposed algorithm that it is automatic and requires no human interaction so therefore, it is better than already existing algorithms. This paper describes the new method for $2 * 2$ iterations which will able to remove the no of iterations, time and excessive noise. Thinning does not work on $2 * 2$ neighbourhood. In this new algorithm is proposed that will remove extra discontinuity and erosion in the output image.
The process is repeated until in that iteration no point is deleted we stop at that point.

[2] In this author describes about the basic concepts of thinning and tells the how the process works. The main idea is how the image is thinnined at background process such that it does not delete the necessary points for image or pattern recognition. He also define the thinning types and their differences. Zhang and Suen algorithm is explained and using this algorithm. Which works on some steps and matches the assumptions for deleting a point and calculate the thinning rate. Author also explained the methodology for both Verilog and MATLAB. Implementation using Verilog and MATLAB is widely explored and lastly results are compared.

[3] In this paper thinning is explained and its application, Disadvantages like distortion, noise and discontinuity is discussed. Types of thinning that are iterative and non iterative which are further divided in sequential and parallel. In sequential pre-determined order is followed and deletion of point will depend upon the (n-1)th iteration or we can say that all the operations performed so far. Algorithms like Zhang and Suen ,Canny edge Detection, optimized iterative algorithm are discussed what are the steps and the methods. Canny edge detection works on the five different steps that are smoothing, finding gradient, non-maximum suppression, hysteresis thresholding and Edge linking. Finally the results are compared and we came to conclusion that optimized iterative algorithm give us the best result which is verified by applying on many of the images.

[4] In this author focuses on the pattern recognition like preprocessing which further include thinning, noise reduction and presents novel rule base system for skeletonising and a formal mathematical algorithm is explained for Zhang and Suen. In which sub iterations are repeated until no more points validate the delete rules .Finally experimental results are compared. After that author came to some observation that creating a new algorithm is not a good choice but adding new features or we can say enhancing the algorithm is far better method.

[5] In this paper author focuses on the thinning process in which image is given as the input thinning is applied on to the image and thinned image of pixel width characters are produced as output. In this Dark point is represented by 1 and white Points are represented as 0.Algorithm results are compared and evaluated on some of the criteria like quality , width of thinned image ,how much the data is reduced, connectivity. Result Shows that Fast parallel algorithm shows the best result, Secondly Pre-processing Algorithm is better than other and Robust parallel thinning algorithm on third and Guo and hall's Parallel on last.

[6] Thinning was firstly defined by Blum in 1962.It works on reducing time, data and transmission. In this paper the problem of loss connectivity is solved. New algorithm is formed results of both are compared. We conclude that completely removal of $2 * 2$ neighbourhood gives as better results .In this new improved algorithm is far better in terms of thickness as compared to Zhang and Suen algorithm. Implementation, test and results are excecuted in $C$ language 


\section{International Journal of Science and Research (IJSR) ISSN (Online): 2319-7064 \\ Index Copernicus Value (2013): 6.14 | Impact Factor (2015): 6.391}

and performance is measured in terms of thinning rate, thinning speed-parallel algorithm is further divided and briefly explained in which $\mathrm{N} 4(\mathrm{p}), \mathrm{Nd}(\mathrm{p})$ and $\mathrm{N} 8(\mathrm{p})$ neighbourhood concept is explained.

[7] In this paper Medical Axis Transformation (MAT) is explained in which how to obtain original image from the thinned is discussed. Problem of having more than one closest point or boundary is solved. Skeletonization we can say that it is a subset of original component and is of two types Pixel based and Non-Pixel based. In pixel based each pixel plays a equal role for giving a thin image but on another hand in non-pixel based one line or one criteria is followed according to which image is got thinned. After the experimental results author got that skeletonization gives us the better results in terms of compactness, ,thinning speed as compared to thinning in which slight irregularities are still there.

[8] In this paper new technique is introduced which is document image and analysis and recognition(DIAR) which helps to extract information to increase knowledge.it is further divided into two parts textual application and graphical textual works on the text represented in the file where as graphical works on the images.A new method is proposed in which binary image is taken as the input and then contour is anlayzed then if the width of image is one pixel then it is thinned otherwise it again repeat the process.

[9] In this paper the problem solved was that still the surrounding pixels was not taken in considerations and new proposed algorithm can be used to thin digits, symbols, letters or characters which may be written in Hindi ,English, Urdu or can say in any of the language. In this rotation method is applied if we conclude that pixel is on boundary we delete that pixel safely this is Pass-1 rule .Outcomes are compared with the ZS and KNP algorithm in terms of thinning time(in milliseconds) and Thinning rate(\%) and proposed algorithm gives us the best result.

\section{Z-S Algorithm}

It is a very proved and well-known algorithm which was proposed by Zhang and Suen algorithm in 1984.It works on Iterative parallel thinning algorithm which is working on 3*3 neighbourhood

\begin{tabular}{|l|l|l|}
\hline P9 & P2 & P3 \\
\hline P8 & P1 & P4 \\
\hline P7 & P6 & P5 \\
\hline
\end{tabular}

Figure 3: ZS 3*3 Neighbourhood

This algorithm works on the two-iterations combined with directional approach.

General Algorithm:-This will result in obtaining the skeleton from the original image. The pixel is deleted if it satisfies the following condition:-
(a) $2 \leq \mathrm{B}(\mathrm{p} 1) \leq 6$
(b) $\mathrm{A}(\mathrm{p} 1)=1$
(c) $\mathrm{p} 2 * \mathrm{p} 4 * \mathrm{p} 6=0$
(d) $\mathrm{p} 4 * \mathrm{p} 6 * \mathrm{p} 8=0$

\section{Conclusion and Result}

Thinning is the important part which take image as the input and give thinned image as the output. It is further divided in to parts. And implemented by various algorithms. Main algorithm is Zhang and Suen algorithm which works on $3 * 3$ neighbourhood which rotate on an image and process the whole image to give as output image. This will help us to reduce complexity, processing time and to filter noise of the image.

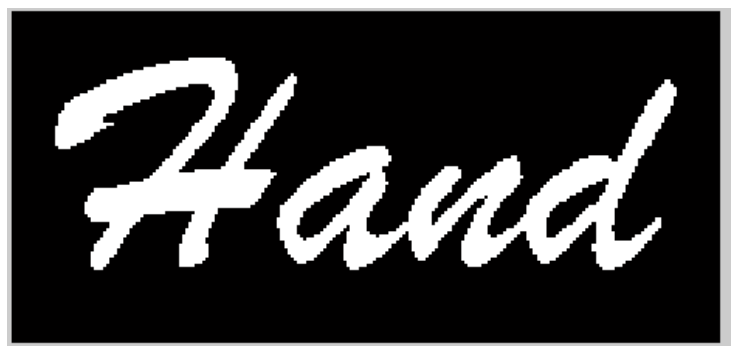

Figure 1: Input Image

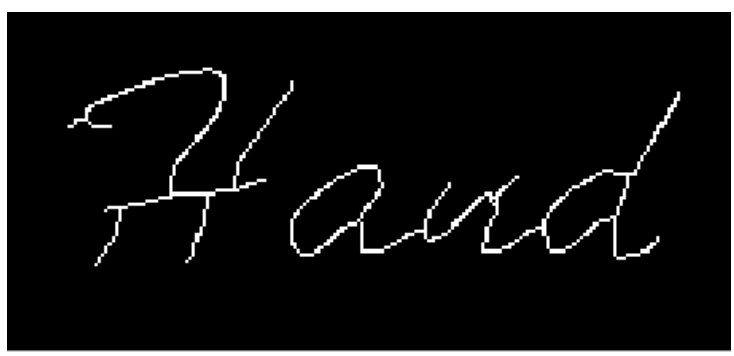

Figure 2: Output Thinned Image

\section{References}

[1] Jagna A. and Kamakshiprasad V,[April 2010] "New parallel binary image thinning algorithm" ARPN Journal of Engineering and Applied sciences

[2] Ashwini S.Karane and S.S. Navalgund ,[2013]'Implementation of an Thinning Algorithm using Verilog and MATLAB" International Journal of current engineering and Technology NCWSE

[3] Miss G.V Padole and Dr S.B Pokle ,[2010]"New Iterative Algorithms for Thinning Binary Images" Electronics and Tele Communication Engineering

[4] RupalK.Snehkunj",[2011] A Comparative Research Of Thinning Algorithm" National Technical Symposium on Advancement computing Technologies

[5] Harish Kumar and Paramjeet Kaur",[2011]A comparative Study Of Iterative Thinning Algorithms for BMP images "International Journal of Computer Science and information Technologies

[6] Lynda Ben Boudaoud and Abderrahmane", [2015]A New Thinning for Binary Images"Institute of Electrical and Electronics Enginners

[7] B.Vanajakshi and Dr. K. Sri Rama Krishna”,[2010]An Analysis of Thinning and Skeletonization for Shape Representation'International Joint Journal Conference on Engineering and Technology

[8] Siti Norul Sheikh Abdullah, Bilal Bataineh and Taraik Abu-Ain",[2013] Skeletonization Algorithm for Binary Images" $4{ }^{\text {th }}$ International Conference on Electrical Engineering and Informatics 


\section{International Journal of Science and Research (IJSR) \\ ISSN (Online): 2319-7064}

Index Copernicus Value (2013): 6.14 | Impact Factor (2015): 6.391

[9] A.Jagna“An efficient independent thinning algorithm",[October2014]International Journal of Advanced Research In Computer and Communication Engineering 